\title{
A search for clusters at high redshift
}

\section{Spectroscopy of $\mathrm{H} \alpha$ emitters in a proto-cluster at $z=2.16$}

\author{
J. D. Kurk ${ }^{1}$, L. Pentericci ${ }^{2}$, R. A. Overzier ${ }^{1}$, H. J. A. Röttgering ${ }^{1}$, and G. K. Miley ${ }^{1}$ \\ 1 Sterrewacht Leiden, PO Box 9513, 2300 RA, Leiden, The Netherlands \\ e-mail: kurk@arcetri.astro.it \\ 2 Max-Planck-Institut für Astronomie, Königstuhl 17, 69117, Heidelberg, Germany
}

Received 10 August 2004 / Accepted 7 October 2004

\begin{abstract}
Radio galaxy PKS 1138-262 is a massive galaxy at $z=2.16$, located in a dense environment. We have found an overdensity of $\operatorname{Ly} \alpha$ emitting galaxies in this field, consistent with a proto-cluster structure associated with the radio galaxy. Recently, we have discovered forty candidate $\mathrm{H} \alpha$ emitters by their excess near infrared narrow band flux. Here, we present infrared spectroscopy of nine of the brightest candidate $\mathrm{H} \alpha$ emitters. All these candidates show an emission line at the expected wavelength. The identification of three of these lines with $\mathrm{H} \alpha$ is confirmed by accompanying [N II] emission. The spectra of the other candidates are consistent with $\mathrm{H} \alpha$ emission at $z \sim 2.15$, one being a QSO as indicated by the broadness of its emission line. The velocity dispersion of the emitters $\left(360 \mathrm{~km} \mathrm{~s}^{-1}\right)$ is significantly smaller than that of the narrow band filter used for their selection $\left(1600 \mathrm{~km} \mathrm{~s}^{-1}\right)$. We therefore conclude that the emitters are associated with the radio galaxy. The star formation rates (SFRs) deduced from the $\mathrm{H} \alpha$ flux are in the range 6-44 $M_{\odot} \mathrm{yr}^{-1}$ and the $S F R$ density observed is 5-10 times higher than in the HDF-N at $z=2.23$. The properties of the narrow emission lines indicate that the emitters are powered by star formation and contain very young $(<100 \mathrm{Myr})$ stellar populations with moderately high metallicities.
\end{abstract}

Key words. galaxies: active - galaxies: clusters: general - galaxies: evolution - cosmology: observations cosmology: early Universe

\section{Introduction}

High redshift clusters are prime subjects for the study of galaxy formation and cosmology. The powerful radio galaxy PKS $1138-262$ at $z=2.156$ appears to be the brightest galaxy in a high redshift cluster. We have discovered an overdensity of Ly $\alpha$ emitters within 1.5 Mpc of 1138-262 (Kurk et al. 2000, Paper I). The redshifts of 14 emitters were spectroscopically confirmed to be in the range $2.14<z<2.18$ (Pentericci et al. 2000, Paper II). In addition, we have carried out near infrared imaging (Kurk et al. 2004, Paper III). The number of $K$ band galaxies and extremely red objects in this field is higher than in blank fields. We found 40 objects with excess narrow band flux, consistent with $\mathrm{H} \alpha$ emission at $z \sim 2.16$. The surface density of $\mathrm{H} \alpha$ emitters increases towards the radio galaxy and their average $K$ magnitude is lower and therefore their inferred stellar mass higher than for the Ly $\alpha$ emitters. Here, we present infrared spectroscopy of nine candidate $\mathrm{H} \alpha$ emitters to confirm their redshift and determine the velocity dispersion of the sample. We assume a flat Universe with $h_{0}=0.65$ and $\Omega_{\mathrm{m}}=0.3$.

\section{Selection, observations and data reduction}

With the aim of detecting $\mathrm{H} \alpha$ emitting galaxies in the protocluster associated with PKS 1138-262, imaging in $K_{\mathrm{s}}$ and in a narrow band filter $\left(\lambda_{\mathrm{c}}=2.07 \mu, F W H M=0.026 \mu\right)$ was carried out, employing two pointings covering a total field of $12.5 \mathrm{arcmin}^{2}$. There are 40 objects with rest frame equivalent width $\left(E W_{0}\right)>25 \AA$ (see Paper III). From the list of 29 candidate $\mathrm{H} \alpha$ emitters within 1.3 from the radio galaxy, we selected those with $\mathrm{H} \alpha$ flux $>3.5 \times 10^{-17} \mathrm{erg} \mathrm{cm}^{-2} \mathrm{~s}^{-1}$. Furthermore, we selected those that were conveniently located for placement in the slit for spectroscopic follow-up, which was carried out with ISAAC at VLT Antu (UT1) ${ }^{1}$. The short wavelength camera of ISAAC is equipped with a Rockwell Hawaii $1024^{2}$ pixel $\mathrm{Hg}: \mathrm{Cd}: \mathrm{Te}$ array which has a projected pixel scale of $0.147^{\prime \prime}$. We used the medium resolution grating in second order resulting in a dispersion of $1.23 \AA$. The observations were carried out in the nights of March 23 and 25, 2002 under variable seeing, which was just below $1^{\prime \prime}$ for most of the time. The $1^{\prime \prime} \times 120^{\prime \prime}$ slit employed resulted in a resolution of $\sim 2600$. During acquisition, the slit was first positioned on a bright point source (a star or the radio galaxy) and subsequently positioned at the midpoint between two candidates, which was always within $32^{\prime \prime}$. We have employed four slit positions, each targeted at two or three candidate emitters for $3.5 \mathrm{~h}$ (3.75 in one case). In total, nine candidates were observed, one of which was included in

1 Based on observations carried out at the European Southern Observatory, Paranal, Chile, project P68.A-0184(A). 


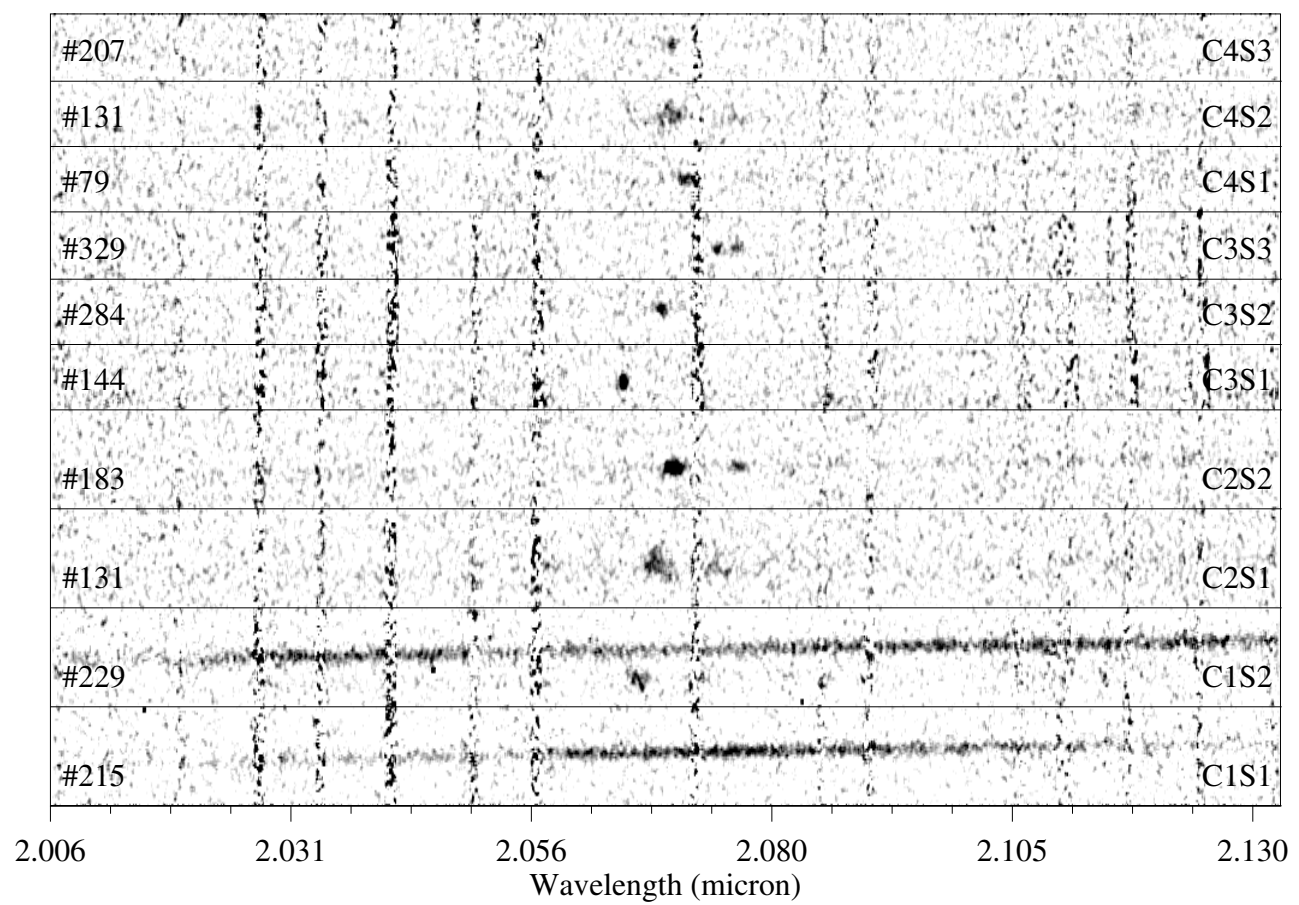

Fig. 1. The ten two dimensional spectra observed through four slits. Skyline residuals are visible as vertical lines with a higher noise level. Slit and spectrum number are indicated, as are the object number from the $\mathrm{H} \alpha$ candidate list. The images have been smoothed with a mask of $3 \times 3$ pixels. Near the emission line of candidate 229 , continuum emission of a serendipitous galaxy is visible. The spectrum of candidate 215 shows a very broad line which almost covers the full spectral range.

two slits. Per slit, we obtained 14 (15) frames of 15 min with offsets of 15,18 or $20^{\prime \prime}$ in ABBA sequence with additional random jitter offsets up to 5,2 , or $1^{\prime \prime}$, respectively, to avoid recurrent registration of spectra on bad pixels. Standard stars were observed with the same slit at a range of airmasses during the nights to correct for telluric absorption and to calibrate the data in flux. All observations were carried out at airmass below 1.8.

Standard data reduction was carried out using pairwise frame subtraction, resulting in a final frame with positive spectra accompanied by negative spectra on both sides. Care was taken during setup to ensure that these negative spectra did not overlap with positive ones. One dimensional spectra of the candidate emitters were extracted from the positive twodimensional spectra using the spatial profile of a standard star spectrum observed during that night and averaged per 3 pixels yielding bins of $3.7 \AA$. The spectral resolution is $7 \AA$, as measured from the $F W H M$ of the skylines. The wavelength calibration is based on the $\mathrm{OH}$ skylines observed. The telluric standards observed at a range of airmasses show only small variations (less than a percent on average). An average absorption spectrum per night was used to correct for telluric absorption. There are no spectrophotometric flux standards in the infrared. We have therefore used one of the telluric standards (Hip043868) with spectral type B1. Such stars have a featureless spectrum in this wavelength region given by a black body curve at $T=25500 \mathrm{~K}$. The curve was normalized to the $K$ magnitude of the star and subsequently used to divide the extracted and absorption corrected spectrum to obtain the flux calibration. The two dimensional spectra of all objects are shown in Fig. 1.

\section{Results}

All candidates observed show an emission line, which means that our selection was $100 \%$ efficient. We have fit Gaussian curves to the emission lines applying a least squares method (see Fig. 2) in order to determine their central wavelength, deconvolved $F W H M$ (if resolved), flux and $E W_{0}$ (Table 1). Also presented in this table is the SFR derived from the $\mathrm{H} \alpha$ and UV luminosities (see discussion in Paper III), which is in the range 6-44 $M_{\odot} \mathrm{yr}^{-1}$. We do not detect continuum emission in most of the spectra, except in the co-added spectrum of candidate 131, 215 and the serendipitous object in slit one $\left(0.6 \pm 0.6,0.7 \pm 0.6\right.$ and $1.7 \pm 0.7 \times 10^{-18} \mathrm{erg} \mathrm{cm}^{-2} \mathrm{~s}^{-1} \AA^{-1}$, respectively), which compare favourably with the line subtracted broad band fluxes measured by imaging in Paper III $(0.6,1.0$ and $2.1 \times 10^{-18} \mathrm{erg} \mathrm{cm}^{-2} \mathrm{~s}^{-1} \AA^{-1}$, respectively). The $E W_{0}$ is therefore based on the line flux measured in the spectra and the broad band magnitude measured on the images.

The spectra of objects 131, 183 and 229 were fit by two Gaussians for which the relative centers were fixed as for $\mathrm{H} \alpha$ and $[\mathrm{N}$ II] $\lambda 6583 \AA$. Candidate 329 shows two emission peaks only $17.8 \pm 2.7 \AA$ apart. Both lines could be $\mathrm{H} \alpha$ emission from one galaxy with two components, seperated by $\sim 250 \mathrm{~km} \mathrm{~s}^{-1}$ in velocity. We have considered the possibility that the lines are due to [O II] emission at $z=4.5674$ which would have a separation of $15.0 \AA$. The emission line ratio of the supposed [O II] $\lambda 3729 / 3726$ would be 0.7 which implies an electron density $\sim 10^{3} \mathrm{~cm}^{-3}$, normally only observed in the central parts of nebulae (Osterbrock 1989). In addition, a faint ( $B=27.0$ ) counterpart in the $B$ band, sampling a wavelength range below $912 \AA$ for $z=4.6$, makes the identification with 


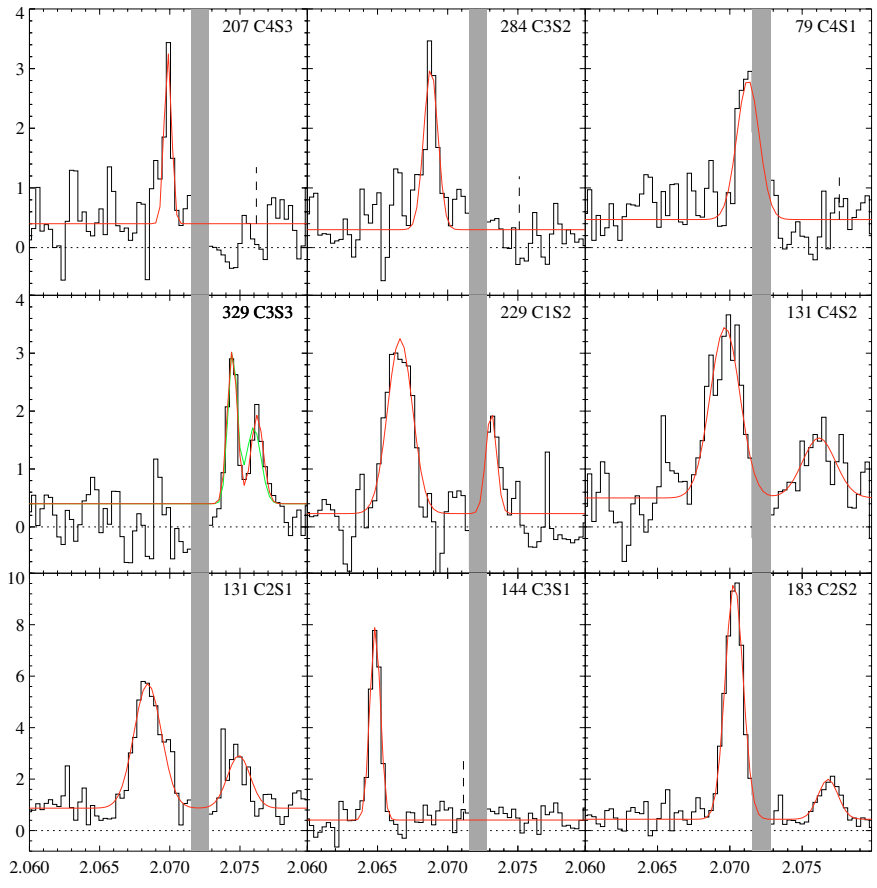

Fig. 2. One dimensional spectra of the eight narrow line emitters (histograms) with fits overlayed (solid lines). Object 329 has also an alternative fit for [O II]. Units are in $10^{-18} \mathrm{erg} \mathrm{cm}^{-2} \mathrm{~s}^{-1} \AA^{-1}$ and $\mu \mathrm{m}$. The grey band denotes the position of a sky line. The dashed line indicates the position of the [N II] line for identification of the detected line with $\mathrm{H} \alpha$. Note the deviant flux density scale in the last row.

[O II] improbable. Candidate 131 was included in two slits, with a position angle difference of $40^{\circ}$. The two fits to the spectra of emitter 131 indicate a velocity difference of $180 \mathrm{~km} \mathrm{~s}^{-1}$ which can be explained by the fact that different regions of the galaxy have been sampled.

For how many of the emission lines can we be sure that the identification with $\mathrm{H} \alpha$ at $z \sim 2.15$ is correct? For the three objects with confirming [N II] lines, we can be certain. For the QSO object 215, [N II] is blended with the very broad $\mathrm{H} \alpha$ line and impossible to discern. Given a $[\mathrm{N}$ II $] / \mathrm{H} \alpha$ line ratio of $1 / 3$, we do not expect to detect the [N II] line for objects 79, 207, 284,329 above the noise and the identification with $\mathrm{H} \alpha$ is therefore consistent but not $100 \%$ certain. An identification with $\mathrm{H} \alpha$ for object 144 can only be true if the $[\mathrm{N} \mathrm{II}] / \mathrm{H} \alpha$ ratio is $<1 / 6$ (object 183 has an observed ratio of $1 / 5.5$ ). An alternative identification with [O III] $\lambda 5007 \AA$ is improbable, as we do not detect its counterpart [O III] $\lambda 4958 \AA$ at $2.045 \mu$. We consider this therefore a probable $\mathrm{H} \alpha$ identification.

At least three of the emission lines are spatially extended. In two cases, we detect unordered velocity structure, but the morphology of the two dimensional spectrum of candidate 229 resembles a rotation curve. A fit to this structure results in a rotation velocity of $50 \mathrm{~km} \mathrm{~s}^{-1}$ at $6 \mathrm{kpc}$ radius, implying a dynamical mass of $3.5 \times 10^{9} M_{\odot}$.

\section{Discussion}

In Fig. 3 we show the redshift distribution of the 9 emitters, assuming that all emission lines can be identified as $\mathrm{H} \alpha$.
For this plot, we used the average redshift of the two lines of object 329. Also plotted is the sensitivity curve of the narrow band filter used to select the candidates. A random distribution of emitter redshifts would follow this curve. We have run a Monte Carlo simulation with 10000 realizations of a randomly sampled distribution of emitters given this filter as selection criterium. Although the mean of the measured distribution is only $0.18 \sigma$ away from the mean of a random sample, its dispersion is much smaller, deviating by $1.75 \sigma$ from a random sample. The probability that the redshifts we measure are drawn from a random distribution is therefore $8 \%$. Note that the redshift of the radio galaxy is 2.156 (Röttgering et al. 1997), very close to the mean of the selection filter (2.152), and to the mean of the measured redshift distribution (2.154). The distribution is consistent with a group of $\mathrm{H} \alpha$ emitters associated with the radio galaxy. The velocity dispersion of this group is $360 \mathrm{~km} \mathrm{~s}^{-1}$ (using the gapper sigma method, Beers et al. 1990), while the virial radius of the nine emitters is $0.45 \mathrm{Mpc}$, implying a virial mass of $8 \times 10^{13} M_{\odot}$ (assuming that all lines can be identified with $\mathrm{H} \alpha$ and taking the mean of the two redshifts for object 131). This mass is merely illustrative as at this redshift it is improbable that the structure is virialized. The velocity dispersion of the $\mathrm{H} \alpha$ emitters is smaller than the velocity dispersion of the confirmed Ly $\alpha$ emitters, both for the complete sample $\left(1050 \mathrm{~km} \mathrm{~s}^{-1}\right)$ and the nine within the solid angle of the two ISAAC fields (760 $\mathrm{km} \mathrm{s}^{-1}$, shaded part of histogram in Fig. 3). There is no evidence for a bimodal redshift distribution as observed for the Ly $\alpha$ emitters.

We can construct a complete sample out of the spectroscopic sample by excluding the two objects with the lowest $\mathrm{H} \alpha$ flux and including the radio galaxy. This collection represents all candidate $\mathrm{H} \alpha$ emitters with $F_{\mathrm{H} \alpha}>4.0 \times$ $10^{-17} \mathrm{erg} \mathrm{cm}^{-2} \mathrm{~s}^{-1}$ within 1.3 from the radio galaxy. The $F W H M$ of the narrow band filter $(2.134<z<2.174)$ and the solid angle given above define a comoving volume of $815 \mathrm{Mpc}^{3}$, resulting in a volume density of $0.010 \mathrm{Mpc}^{-3}$, which is a factor four higher than the density of confirmed Ly $\alpha$ emitters in this field. All star forming objects detected have line fluxes lower than the high redshift $\mathrm{H} \alpha$ surveys discussed in Paper III, but we can compare the SFR density to the density at $z=2.23$ derived from $\mathrm{H} \alpha$ emission in the HDF-N as measured by Iwamuro et al. (2000). Following their cosmology $\left(h_{0}=0.5\right.$, $\left.q_{0}=0.5\right)$ and procedure to correct for the part of the H $\alpha$ luminosity function below the detection limit, we obtain a $S F R$ density of $0.48 M_{\odot} \mathrm{yr}^{-1} \mathrm{Mpc}^{-3}$. This is 10 (5) times higher than the (reddening corrected) value obtained for the HDF-N. Using the redshift range defined by the $\mathrm{H} \alpha$ emitters $(2.146<z<2.164)$ results in values that are larger by a factor of two. Likewise, smaller SFR densities would result if some of the $\mathrm{H} \alpha$ lines have been misidentified.

The properties of the detected emission lines provide information about the physical conditions in the galaxies. The $F W H M$ of the narrow nebular emission lines detected are in the range $40-360 \mathrm{~km} \mathrm{~s}^{-1}$ with an average of $190 \mathrm{~km} \mathrm{~s}^{-1}$. These values are comparable to those found for LBGs at $z \sim 3$ by Pettini et al. (2001). The [N II]/H $\alpha$ ratio can be used to distinguish narrow-line active galaxies from H II region-like galaxies (Veilleux \& Osterbrock 1987). The three emitters with 
Table 1. Properties of the observed emission lines.

\begin{tabular}{|c|c|c|c|c|c|c|c|c|c|c|c|}
\hline \multirow{2}{*}{$\begin{array}{l}\text { ID } \\
(1)\end{array}$} & \multirow{2}{*}{$\begin{array}{c}S \\
(2)\end{array}$} & \multirow{2}{*}{$\begin{array}{l}\text { RA and Dec (J2000) } \\
(3)\end{array}$} & \multirow{2}{*}{$\begin{array}{l}\text { Line } \\
(4)\end{array}$} & \multirow{2}{*}{$\begin{array}{c}\text { Fit } \\
(5,6)\end{array}$} & \multirow{2}{*}{$\begin{array}{c}z \\
(7)\end{array}$} & \multirow{2}{*}{$\begin{array}{c}F W H M \\
(8)\end{array}$} & \multirow{2}{*}{$\begin{array}{l}\text { Flux } \\
(9)\end{array}$} & \multirow{2}{*}{$\begin{array}{r}E W_{0} \\
(10) \\
\end{array}$} & \multicolumn{2}{|c|}{${ }_{\mathrm{H} \alpha} S F R_{\mathrm{UV}}$} & \multirow{2}{*}{$\begin{array}{l}\mathrm{O} / \mathrm{H} \\
(13)\end{array}$} \\
\hline & & & & & & & & & (11) & (12) & \\
\hline 79 & 4 & $11: 40: 52.62-26: 30: 01.0$ & $\mathrm{H} \alpha$ & 1,2 & 2.1558 & $160 \pm 80$ & $3.6 \pm 1.9$ & $90 \pm 50$ & $11 \pm 6$ & $33 \pm 1$ & \\
\hline \multirow[t]{2}{*}{131} & 2 & $11: 40: 51.28-26: 29: 38.7$ & $\mathrm{H} \alpha$ & 3,1 & 2.1518 & $320 \pm 50$ & $12.3 \pm 2.2$ & $65 \pm 15$ & $39 \pm 7$ & $19 \pm 1$ & 8.8 \\
\hline & & & {$[\mathrm{N}$ II $]$} & & & $270 \pm 100$ & $4.4 \pm 2.0$ & $25 \pm 10$ & & & \pm 0.2 \\
\hline \multirow[t]{2}{*}{131} & 4 & $11: 40: 51.28-26: 29: 38.7$ & $\mathrm{H} \alpha$ & 3,1 & 2.1537 & $360 \pm 80$ & $8.2 \pm 2.4$ & $45 \pm 15$ & $26 \pm 8$ & $19 \pm 1$ & 8.8 \\
\hline & & & {$[\mathrm{N}$ II $]$} & & & $420 \pm 250$ & $3.4 \pm 2.5$ & $20 \pm 15$ & & & \pm 0.2 \\
\hline 144 & 3 & $11: 40: 43.45-26: 29: 37.5$ & $\mathrm{H} \alpha$ & 1,3 & 2.1463 & $40 \pm 20$ & $6.7 \pm 1.3$ & $330 \pm 70$ & $21 \pm 4$ & $15 \pm 1$ & \\
\hline \multirow[t]{2}{*}{183} & 2 & $11: 40: 46.15-26: 29: 24.9$ & $\mathrm{H} \alpha$ & 3,1 & 2.1546 & $170 \pm 20$ & $13.8 \pm 1.7$ & $230 \pm 35$ & $44 \pm 5$ & $40 \pm 1$ & 8.6 \\
\hline & & & {$[\mathrm{N}$ II $]$} & & & $200 \pm 120$ & $2.5 \pm 1.7$ & $40 \pm 30$ & & & \pm 0.4 \\
\hline 207 & 4 & $11: 40: 50.20-26: 29: 21.0$ & $\mathrm{H} \alpha$ & 1,2 & 2.1540 & $<100$ & $1.9 \pm 0.9$ & $50 \pm 25$ & $6 \pm 3$ & $12 \pm 1$ & \\
\hline 215 & 1 & $11: 40: 46.01-26: 29: 16.9$ & $\mathrm{H} \alpha$ & 3,1 & 2.1568 & $5300 \pm 800$ & $46.2 \pm 8.8$ & $150 \pm 30$ & $\dagger$ & & \\
\hline \multirow[t]{2}{*}{229} & 1 & $11: 40: 46.10-26: 29: 11.5$ & $\mathrm{H} \alpha$ & 3,1 & 2.1489 & $290 \pm 60$ & $7.1 \pm 1.9$ & $30 \pm 10$ & $23 \pm 6$ & $26 \pm 1$ & 8.8 \\
\hline & & & {$[\mathrm{N}$ II $]$} & & & $130 \pm 90$ & $2.4 \pm 1.7$ & $11 \pm 8$ & & & \pm 0.3 \\
\hline 284 & 3 & $11: 40: 45.58-26: 29: 02.4$ & $\mathrm{H} \alpha$ & 1,2 & 2.1523 & $90 \pm 50$ & $3.0 \pm 1.4$ & $300 \pm 140$ & $10 \pm 4$ & $6 \pm 1$ & \\
\hline \multirow[t]{2}{*}{329} & 3 & $11: 40: 46.88-26: 28: 41.4$ & $\mathrm{H} \alpha$ & 2,3 & 2.1609 & $60 \pm 40$ & $2.7 \pm 1.3$ & $1350 \pm 700$ & $9 \pm 4$ & $7 \pm 1$ & \\
\hline & & & $\mathrm{H} \alpha$ & 2,3 & 2.1636 & $110 \pm 30$ & $2.0 \pm 1.1$ & $1000 \pm 550$ & $6 \pm 4$ & & \\
\hline
\end{tabular}

Notes: (1) Candidate number; (2) slit number; (3) coordinates; (4) line identification; (5) type of fit: 1) one Gaussian curve; 2) two Gaussian curves; 3) two Gaussians for $\mathrm{H} \alpha$ and $[\mathrm{NII}]$; (6) identification is 1) certain, detection of [N II]; 2) consistent, expected non detection of [N II]; 3) possible; (7) redshift with random error 0.0002, except for candidate 215 , for which it is 0.002 ; (8) $F W H M$ in $\mathrm{km} \mathrm{s}^{-1}$; (9) flux in $10^{-17} \mathrm{erg} \mathrm{cm}^{-2} \mathrm{~s}^{-1}$; (10) $E W_{0}$ in $\AA$; (11) $S F R_{\mathrm{H} \alpha}$ in $M_{\odot} \mathrm{yr}^{-1}$; (12) $S F R_{\mathrm{UV}}$ in $M_{\odot} \mathrm{yr}^{-1}$; (13) metallicity in $12+\log [\mathrm{O} / \mathrm{H}]\left(^{\dagger}\right) \mathrm{H} \alpha$ unrelated to $S F R$.

detected $[\mathrm{N}$ II] have $-0.74<\log ([\mathrm{N}$ II $] / \mathrm{H} \alpha)<-0.42$, which puts them among the star forming galaxies. In the absence of shock excitation, the $[\mathrm{N} \mathrm{II}] / \mathrm{H} \alpha$ ratio can also be used as metallicity indicator. Using the empirical relation calibrated by Denicoló et al. (2002), the average ratio of the three emitters implies $12+\log (\mathrm{O} / \mathrm{H}) \approx 8.7$. This value is comparable to the broad range of values obtained for present-day spiral galaxies (van Zee et al. 1998). The $E W_{0}$ of some detected narrow lines are surprisingly high, up to $1350 \AA$. This can be explained by very young stellar populations where the continuum radiation around $6000 \AA$ is still very weak. $E W_{0}$ values between 200 and $330 \AA$ imply an age $<100 \mathrm{Myr}$ (Leitherer et al. 1999). The moderately high metallicities found for the objects with detected [N II] emission, however, require that the galaxies are near the end of the star formation event. This requirement seems to indicate that these emitters have undergone a very similar evolution.

\section{Conclusion}

Infrared spectroscopy has established the presence of nine line emitters within 0.6 Mpc of the HzRG PKS 1138-262. Three emitters show an additional line which confirms the identification with $\mathrm{H} \alpha$ at $z=2.15$, while four more have spectra consistent with $\mathrm{H} \alpha$ at this redshift, one being a QSO as indicated by the broadness of its emission line. One emitter shows only a single strong line, which is possibly $\mathrm{H} \alpha$ and one emitter exhibits two lines which probably originate from two emission

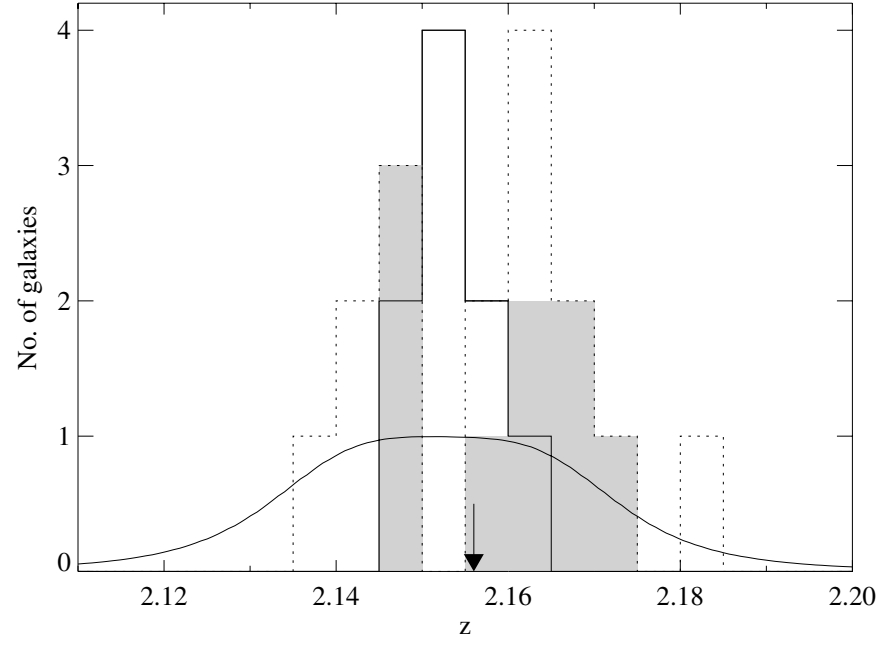

Fig. 3. Redshift histogram for the $\mathrm{H} \alpha$ emitters (solid) and the previously known Ly $\alpha$ emitters (dotted, and shaded for those within ISAAC field). An arrow denotes the redshift of 1138-262. Also shown is the sensitivity curve of the narrow band filter used for the selection of the $\mathrm{H} \alpha$ candidates. The surface beneath the curve is normalized to nine.

line regions within one galaxy at $z=2.16$. Additional evidence for identification of all observed lines with $\mathrm{H} \alpha$ is the small velocity dispersion $\left(360 \mathrm{~km} \mathrm{~s}^{-1}\right)$ as compared with the width of the selection filter. This dispersion is also smaller than the dispersion of the Ly $\alpha$ emitters. The star formation rate density 
of the observed emitters is a factor ten higher than found at $z=2.23$ in the HDF-N. These results support the formerly advocated ideas that PKS 1138-262 is located in a proto-cluster at $z=2.16$. The properties of the narrow emission lines indicate that the emitters are powered by star formation and contain very young $(<100 \mathrm{Myr})$ stellar populations with moderately high metallicities. It seems that we observe these galaxies near the end of their first and major burst of star formation.

Acknowledgements. We are grateful to the ESO VLT staff for excellent support during the observing run. We acknowledge fruitful discussions with B. Venemans and S. di Serego Alighieri. Comments of the anonymous referee have also helped to improve the manuscript. This research has made use of the NASA/IPAC Extragalactic Database (NED) which is operated by the Jet Propulsion Laboratory, California Institute of Technology, under contract with the National Aeronautics and Space Administration. We have also made use of NASA's Astrophysics Data System Bibliographic Services.

\section{References}

Beers, T. C., Flynn, K., \& Gebhardt, K. 1990, AJ, 100, 32

Denicoló, G., Terlevich, R., \& Terlevich, E. 2002, MNRAS, 330, 69

Iwamuro, F., Motohara, K., Maihara, T., et al. 2000, PASJ, 52, 73

Kurk, J., Pentericci, L., Röttgering, H., \& Miley, G. 2004, A\&A, 428, 793

Kurk, J., Röttgering, H., Pentericci, L., et al. 2000, A\&A, 358, L1

Leitherer, C., Schaerer, D., Goldader, J. D., et al. 1999, ApJS, 123, 3

Osterbrock, D. E. 1989, Astrophysics of Gaseous Nebulae and Active Galactic Nuclei (Mill Valley, CA, USA: University Science Books)

Pentericci, L., Kurk, J., Röttgering, H., et al. 2000, A\&A, 361, L25

Pettini, M., Shapley, A. E., Steidel, C. C., et al. 2001, ApJ, 554, 981

Röttgering, H. J. A., van Ojik, R., Miley, G. K., et al. 1997, A\&A, 326,505

van Zee, L., Salzer, J. J., Haynes, M. P., O’Donoghue, A. A., \& Balonek, T. J. 1998, AJ, 116, 2805

Veilleux, S., \& Osterbrock, D. E. 1987, ApJS, 63, 295 